\title{
Ocorrência de Cryptosporidium sp em amostras fecais de crianças, menores de 10 anos de idade, com indicação clínica de Rotavírus
}

\author{
Occurrence of Cryptosporidium sp in fecal samples of children \\ less than 10 years old with clinical indication of Rotavirus \\ Sidnei da Silva1, Siudomar Pereira da Silva ${ }^{2}$, Yára de Souza Gouveia ${ }^{1}$, Nívia de Oliveira da Silva ${ }^{2,3}$, \\ Márcia Elizabeth Reis Monteiro de Melo, ${ }^{2,4}$, Hércules Moura1, Renata Heisler Neves', \\ Alexandre Ribeiro Bello e José Roberto Machado-Silva'
}

\begin{abstract}
Resumo A partir da indicação clínica de Rotavírus em fezes de 485 crianças, investigou-se a presença de oocistos de Cryptosporidium sp. Não houve diferenças significativas entre a positividade de Cryptosporidium $\mathrm{sp}$ e rotavírus com a consistência das fezes. Cryptosporidium sp deve ser incluído na investigação diagnóstica dos quadros diarréicos em crianças.
\end{abstract}

Palavras-chaves: Cryptosporidium sp. Rotavírus. Crianças.

Abstract The presence of oocysts of Cryptosporidium sp was investigated in 485 fecal samples of children with clinical indication of Rotavirus. No significant differences were observed between Cryptosporidium $\mathrm{sp}$. and rotavirus occurrence and fecal consistency. Cryptosporidium sp also should be performed in the laboratory diagnosis of diarrheic episodes in children.

Key-words: Cryptosporidium sp. Rotavirus. Children.

As gastroenterites são as principais causas de mortalidade infantil em países desenvolvidos e em desenvolvimento ${ }^{11}$. Distintos agentes etiológicos podem levar a este quadro onde Rotavírus é o principal agente viral ${ }^{7}$. Cryptosporidium sp está relacionado com surtos de diarréia, após ingestão de água ou alimentos contaminados com oocistos (water food-borne outbreaks) ${ }^{9}{ }^{12}$. Recentemente, foram encontrados oocistos de Cryptosporidium sp em represas contaminadas por esgoto no município de Campinas (São Paulo, Brasil) ${ }^{4}$. Neste artigo, relata-se a ocorrência de Cryptosporidium sp em amostras fecais de crianças com indicação clínica de Rotavírus.

Entre setembro de 1999 e setembro de 2000, 485 amostras de fezes de crianças (abaixo de 10 anos de idade) com diarréia ou história prévia de quadro diarréico no Município do Rio de Janeiro, foram submetidas à pesquisa de Rotavírus (aglutinação pelo látex SLIDEX ROTA KIT ${ }^{\mathrm{R}}$ ). Estas amostras foram concentradas pelo método de Ritchie modificado e coradas pelas técnicas da safranina a quente ${ }^{2} \mathrm{e}$ Kinyoun modificado a frio para a pesquisa oocistos de Cryptosporidium sp. Para tal, dois observadores fizeram a leitura de todos os campos das lâminas por microscopia de campo claro em objetiva de imersão (1000X). Os dados foram submetidos ao teste do quiquadrado, considerando-se diferenças significativas, valores de $p<0,05$.

Das 485 amostras, $33(6,8 \%)$ foram positivas para Rotavírus e $16(3,3 \%)$ para Cryptosporidium sp.

\footnotetext{
1. Departamento de Patologia e Laboratórios da Faculdade de Ciências Médicas da Universidade do Estado do Rio de Janeiro, Rio de Janeiro, RJ. 2. Laboratório de Parasitologia do Hospital Universitário Pedro Ernesto da Universidade do Estado do Rio de Janeiro, Rio de Janeiro, RJ. 3. Laboratórios Médicos Dr. Sérgio Franco, Rio de Janeiro, RJ. 4. Laboratório Diagnósticos da América, Rio de Janeiro, RJ.

Apoio financeiro: UERJ

Endereço para correspondência: Dr. José Roberto Machado e Silva. Dept ${ }^{\circ}$ de Patologia e Laboratórios/FCM/UERJ. R. Prof. Manuel de Abreu 444/5 andar, Vila Isabel, 20550-170 Rio de Janeiro, RJ, Brasil.

Tel: 5521 2587-6148, Fax: 5521 2587-6112

e-mail:machado@uerj.br

Recebido em 10/10/2001

Aceito em 24/2/2003
} 
O tamanho limitado das amostras positivas para Cryptosporidium sp não permitiu uma comparação entre a especificidade e sensibilidade dos métodos. Os oocistos de Cryptosporidium sp coraram-se de maneira homogênea e regular pelas duas técnicas. O maior número de casos de Rotavírus foi em crianças na faixa de 1 ano de idade (12,3\%). Entre os meses de junho e agosto (Tabela 1), este vírus apresentou maior ocorrência (13,8\%), confirmando a sua sazonalidade ${ }^{3}$. Esta diferiu de Cryptosporidium sp com maior número de casos entre dezembro e fevereiro (5,6\%), em acordo com outros achados 5 .

A presença de Cryptosporidium sp tem sido descrita em áreas favelizadas e populações atendidas em ambiente hospitalar ${ }^{51011}$. A população-alvo (crianças abaixo de 10 anos de idade) aqui estudada, recorrera a laboratórios de medicina laboratorial da rede privada para pesquisa de Rotavírus. Em crianças atendidas (0 a 12 anos de idade) em Uberlândia (MG), a prevalência de Cryptosporidium sp foi de $4,2 \%{ }^{5}$. No presente trabalho, a prevalência $(3,3 \%)$ foi inferior a estes dados mas, superiores aos relatados $(1,8 \%)$ em crianças na cidade de Ribeirão Preto $(\mathrm{SP})^{10}$. No município de São Paulo, crianças até cinco anos de idade apresentaram uma prevalência de $17,4 \%^{8}$.

A consistência das amostras fecais e a positividade dos patógenos não foram significativamente diferentes $(p>0,05)$. A infecção por Cryptosporidium sp está freqüentemente associada com episódios diarréicos, persistentes, com duração variável ${ }^{11}$. No presente trabalho, este enteropatógeno foi encontrado, indistintamente, em fezes diarréicas e pastosas, exceto em fezes moldadas (Tabela 2), sem que houvesse diferença estatística, entre elas. Ainda que não tenha

\begin{tabular}{|c|c|c|c|c|c|}
\hline \multirow[b]{2}{*}{ Meses } & \multirow{2}{*}{$\begin{array}{c}\text { Amostras } \\
n^{\circ}\end{array}$} & \multicolumn{2}{|c|}{ Rotavírus } & \multicolumn{2}{|c|}{ Cryptosporidium sp } \\
\hline & & $\mathrm{n}^{\circ}$ & $\%$ & $\mathrm{n}^{\circ}$ & $\%$ \\
\hline Agosto & 22 & 3 & 13,6 & 1 & 4,5 \\
\hline Setembro - novembro & 125 & 7 & 5,6 & 2 & 1,6 \\
\hline Dezembro - fevereiro & 88 & 0 & 0 & 5 & 5,6 \\
\hline Março - maio & 112 & 8 & 7,1 & 5 & 4,4 \\
\hline Junho - agosto & 94 & 13 & 13,8 & 2 & 2,1 \\
\hline Setembro & 44 & 2 & 4,5 & 1 & 2,2 \\
\hline Total & 485 & 33 & 6,8 & 16 & 3,3 \\
\hline
\end{tabular}

Tabela 2 - Distribuição dos casos positivos de Cryptosporidium sp. e Rotavírus, de acordo com a consistência das fezes de criancas (abaixo de 10 anos de idade), entre agosto de 1999 e setembro de 2000, na cidade do Rio de Janeiro, Brasil.

\begin{tabular}{lcrrrrr}
\hline & Amostras & \multicolumn{2}{c}{ Rotavírus } & & \multicolumn{2}{c}{ Cryptosporidium $s p$} \\
\cline { 7 - 8 } \cline { 7 - 8 } Consistência & $\mathrm{N}$ & $\mathrm{n}^{\circ}$ & $\%$ & & $\mathrm{n}^{\circ}$ & $\%$ \\
\hline Líquidas & 102 & 9 & 8,8 & & 6 & 5,8 \\
Pastosas & 317 & 21 & 6,6 & & 10 & 3,1 \\
Moldadas & 66 & 3 & 4,5 & & 0 & 0,0 \\
\hline
\end{tabular}

sido possível estabelecer o período de duração do episódio diarréico, é lícito supor que, sobretudo, nesta fase haja intensa eliminação de oocistos nas fezes. Os dados aqui expressos, indicam que esta excreção não deve cessar nos períodos subseqüentes a este quadro.
No presente levantamento, não foi encontrada concomitância entre Cryptosporidium sp e Rotavírus, como antes descrito ${ }^{6}$. Sugerimos a inserção da pesquisa de oocistos de Cryptosporidium sp, na investigação diagnóstica dos quadros diarréicos em crianças.

\section{REFERÊNCIAS BIBLIOGRÁFICAS}

1. Alverca VO, Gomes TS, Silva MLR, Domingues ALS, Santos NOS. Incidência de gastrenterite infantil de etiologia viral em Juiz de Fora, Minas Gerais, no período de janeiro a dezembro de 1998. Jornal Brasileiro de Patologia 4: 219-227, 2000.

2. Baxby D, Blundell N, Hart CA. The development and performance of a simple, sensitive method for detection the Cryptosporidium oocysts in faeces. Journal of Hygiene 92: 317323, 1984
3. Bittencourt JAF, Arbo E, Malysz AS, Oravec R, Dias C. Seasonal and age distribution of rotavirus infection in Porto Alegre - Brazil. The Brazilian Journal of Infectious Diseases 4: 279-283, 2000.

4. Franco RMG, Fernandes RR, Neto C. Ocurrence of Cryptosporidium oocysts and Giardia cysts in raw water from the Atibaia River, Campinas, Brazil. Revista do Instituto de Medicina Tropical de São Paulo 43: 109-111, 2001. 
5. Gennari-Cardoso ML, Costa-Cruz JM, Castro E, Lima LMFS, Prudente DV. Cryptosporidium $s p$ in children suffering from acute diarrhea at Uberlândia city, State of Minas Gerais, Brazil. Memórias do Instituto Oswaldo Cruz 91:551-554, 1996.

6. Gusmão RHP, Mascarenhas JDP, Gabbay YB, Lins-Lainson Z, Ramos FLP, Monteiro TAF, Valente SA, Linhares AC. Rotaviruses as a cause of nosocomial, infantile diarrhoea in northern Brazil: pilot study. Memórias do Instituto Oswaldo Cruz 90:743-749, 1995.

7. Linhares AC. Rotavirus infection in Brazil: epidemiology, immunity, and potential vaccination. The Brazilian Journal of Infectious Diseases 1: 284-293, 1997.

8. Mangini ACS, Dias RMDS, Grisi SJFE, Escobar AMU, Torres DMAGV, Zuba IPR, Quadros CMS, Chieffi PP. Parasitismo por Cryptosporidium $s p$. em crianças com diarréia aguda.
Revista do Instituto de Medicina Tropical de São Paulo 34: 341-345, 1992.

9. Marshall MM, Naumovitz D, Ortega $\mathrm{Y}$, Sterling $\mathrm{CH}$. Waterborne protozoan pathogens. Clinical Microbiology Reviews 10: 67-85, 1997.

10. Medeiros MIC, Neme SN, Silva P, Capuano DM, Errera MC, Fernandes AS, Valle GR, Avila FA. Etiology of acute diarrhea among children in Ribeirão Preto, Brazil. Revista do Instituto de Medicina Tropical de São Paulo 43: 21-24, 2001.

11. Newman RD, Sears CL, Moore SR, Nataro JP, Wuhib T, Agnew DA, Guerrant RL, Lima AAM. Longitudinal study of Cryptosporidium infection in children in northeastern Brazil. The Journal of Infectious Diseases 180: 167-175, 1999.

12. Nichols GL. Food-borne protozoa. British Medical Bulletin 56: 209-35, 2000. 